\title{
ASSESSING THE USEFULNESS OF THE QUASI-IDEAL OBSERVER FORQUALITY CONTROL IN FLUOROSCOPY
}

Erik Tesselaar and Michael Sandborg

\section{Linköping University Post Print}

\section{Tweet}

N.B.: When citing this work, cite the original article.

Original Publication:

Erik Tesselaar and Michael Sandborg, ASSESSING THE USEFULNESS OF THE QUASIIDEAL OBSERVER FORQUALITY CONTROL IN FLUOROSCOPY, 2015, Radiation Protection Dosimetry.

http://dx.doi.org/10.1093/rpd/ncv434

Copyright: Oxford University Press (OUP): Policy B - Oxford Open Option A http://www.oxfordjournals.org/

Postprint available at: Linköping University Electronic Press

http://urn.kb.se/resolve?urn=urn:nbn:se:liu:diva-123020 


\section{Assessing the usefulness of the quasi-ideal observer for quality control in fluoroscopy}

E Tesselaar ${ }^{1}$ and $M$ Sandborg ${ }^{2}$

${ }^{1}$ Medical Radiation Physics, County Council of Östergötland, Linköping University, SE58185 Linköping, Sweden

${ }^{2}$ Medical Radiation Physics, Department of Medical and Health Sciences and Center for Medical Image Science and Visualisation, Linköping University, SE-58185 Linköping, Sweden

\section{Corresponding author}

Erik Tesselaar

Medical Radiation Physics

County Council of Östergötland

Linköping University

SE-58183 Linköping

Sweden

tel. +46101038059

e-mail: erik.tesselaar@liu.se

\section{Short running title}

Quasi-ideal observer for quality control 
OXMI2015-026

\section{Assessing the usefulness of the quasi-ideal observer for quality control in}

\section{fluoroscopy}

\section{E Tesselaar and M Sandborg}

\section{ABSTRACT}

The aim of this work was to evaluate the reliability of the square of the signal-to-noise ratio rate, $S N R_{\text {rate }}^{2}$ as a precise measurement for quality control test in a digital fluoroscopy system.

The quasi-ideal model observer was used to measure $S N R_{\text {rate }}^{2}$. The dose rate, pulse rate and field of view were varied and their effect on dose efficiency, defined as $S N R_{\text {rate }}^{2} / P_{K A, \text { rate }}$ was evaluated (where $P_{K A \text {,rate }}$ is the air kerma-area product rate). Measurements were repeated to assess reproducibility.

The relative standard deviation in $S N R_{\text {rate }}^{2} / P_{K A \text {,rate }}$ over seven consecutive measurements was $5 \%$. No significant variation in $S N R_{\text {rate }}^{2} / P_{K A, \text { rate }}$ was observed across different pulse rates (10-30 pulses/s). The low-dose rate setting had a superior dose efficiency compared to the medium and high dose rate settings. A smaller field of view resulted in higher dose efficiency.

Our results showed that $S N R_{\text {rate }}^{2} / P_{K A, \text { rate }}$ measurements offer the high precision required in quality control constancy tests. 
OXMI2015-026

\section{INTRODUCTION}

Detectability of low contrast detail is recognised as an important image quality measure in fluoroscopy. It is important in equipment design, in the performance specification of x-ray systems, in acceptance or quality control testing and in dose optimisation. To be useful, measurements of low-contrast detectability or low-contrast visibility need to be sensitive enough to detect subtle changes in image quality. They also need to be precise (i.e. show a limited range of results for identical conditions), as well as accurate and reasonably quick to perform.

Threshold contrast, $\mathrm{C}_{\text {th }}$, and multiple alternative forced choice, MAFC, measurements are commonly used to assess low-contrast detectability with human observers ${ }^{(1,2)}$. With the threshold contrast method, the human observer's task is to assess a range of low-contrast details in varying thicknesses, and to identify the faintest detail they can detect without guessing. This method typically suffers from a large inter- and intra-observer variance, as there is no unique threshold for detection that can easily be described and maintained in a group of human observers. As a consequence, only large changes in image quality can be expected to be statistically significant. The threshold contrast method is, however, simple and quick to set up and evaluate. MAFC measurements, on the other hand, are typically more time consuming to set up and evaluate. The low contrast details need to be just barely detectable for the method to work. However, MAFC measurements are accurate because they measure the true detection performance by the individual human observer. Another problem with human observers is the inter-observer reproducibility, as different observers may vary in their ability to detect low-contrast details. Tapiovaara and Sandborg showed that if only one or two human observers are used to assess image quality in fluoroscopy, only exceptionally large changes in image quality can reliably be detected ${ }^{(3)}$. Therefore, an impractically large number 
of observers are required for a precise assessment using $\mathrm{C}_{\text {th }}$ or MAFC in fluoroscopy. Thilander-Klang et al. showed that absolute assessment of visibility of low-contrast detail images in computed tomography is not reliable ${ }^{(4)}$. Relative assessment (i.e. pairwise comparisons) results in a larger probability of achieving a given proportion of correctly identified changes in low-contrast detail visibility in CT. With only a single observer, however, a change in image quality corresponding to a tube current of at least 50\% was required in order to correctly identify the same proportion of changes with certainty ${ }^{(4)}$.

Due to the limitations of human observers in this context, an objective, direct measurement of low contrast detectability is an attractive alternative for quality control measurements. A channelized Hotelling model observer has previously been used to assess the low-contrast detectability in images of an anthropomorphic phantom in computed tomography ${ }^{(5,6)}$. These studies showed a positive correlation and agreement with human observers in terms of percent correct in 4AFC experiments, and the mathematical model observer was shown to be less time consuming than human observers when performing receiver operating characteristics (ROC) studies.

The square of the signal-to-noise ratio rate $\left(\mathrm{SNR}^{2}\right.$ rate $)$ measures the accumulating rate of information in the fluoroscopy images in an objective and absolute manner. Contrary to ROC and MAFC studies where the pathology or contrast detail must be close to the visibility threshold for the evaluation method to work properly (i.e. non-trivial task), the contrast detail in $\mathrm{SNR}^{2}$ rate measurement can be large to improve its accuracy and precision ${ }^{(3)}$. It has also been shown that the sensitivity of the method is better than methods relying on human observers ${ }^{(3)}$. However, in these previous studies, $\mathrm{SNR}^{2}$ rate measurements were made on an X-ray image intensifier system. The aim of this work was to measure $\mathrm{SNR}^{2}$ rate on a modern fluoroscopy 
OXMI2015-026

system with a flat-panel image detector, and to assess its potential for both quality control measurements and identifying dose-efficient imaging configurations.

\section{MATERIALS AND METHOD}

\section{Imaging equipment}

A Siemens Axiom Artis Zee MP fluoroscopy unit (Siemens Healthcare GmbH, Erlangen, Germany) was used. It is equipped with three different dose rate settings, a range of pulsed fluoroscopy settings and dynamic additional copper filtration. It has a built-in air kerma area product, $\mathrm{P}_{\mathrm{KA}}$-meter (Diamentor, PTW, Freiburg, Germany) that integrates the air kerma over the $\mathrm{x}$-ray beam. The reading from the $\mathrm{P}_{\mathrm{KA}}$-meter, located in the collimator housing, was traceable to the Swedish secondary standards laboratory at the Swedish Radiation Safety Authority in Stockholm ${ }^{(7)}$.

\section{Test objects and image acquisition}

The test phantom consisted of two parts. The homogeneous part consisted of a $2 \mathrm{~mm}$ copper filter (99.9\% Cu, Cambridge Ltd, Huntingdon, England) positioned on top of the collimator under the patient couch. The inhomogeneous part consisted of two layers - first a homogeneous slab of polymethyl methacrylate (PMMA), with an area of $30 \times 30 \mathrm{~cm}^{2}$ and thickness of $40 \mathrm{~mm}$. The second layer was replaceable, either a $10 \mathrm{~mm}$-thick PMMA layer (homogeneous) or a 5 mm-thick aluminium disk, used as contrast detail (inhomogeneous). The patient couch was positioned at the centre of rotation, with the mattress removed, and the PMMA-phantom was placed on top of the couch. The inhomogeneous part of the phantom was placed closer to the image detector than the homogeneous part. The focal-image detector 
distance was $100 \mathrm{~cm}$, the beam area was $20 \times 20 \mathrm{~cm}^{2}$ and the rectangular shaped automatic exposure control chamber in the centre was selected. Subsequently, 1024 consecutive frames of fluoroscopy images were acquired and stored. These image frames were then exported for further image analysis.

Two series of image frames were acquired, one with the contrast details present in the phantom (signal images), and the other without the contrasting details present (background images). Since the contrasting details were small (with respect to the $\mathrm{x}$-ray beam area), the automatic exposure control setting was not affected by the contrasting details in the phantom. The image acquisition parameters (e.g. tube voltage, tube current, pulse time, added filtration etc.) were therefore the same between the two acquisitions. With a pulse rate of $15 \mathrm{~s}^{-1}$, the time to acquire the 1024 images in each acquisition series was 68 s. A Matlab script (The MathWorks, Natick, USA) was used to select a region of interest of $64^{2}$ pixels around the contrast details and to convert DICOM image files to the proprietary format used in the software FluoroQuality v.2.0 $0^{(8)}$. Figure 1 shows examples of single signal, single background images, and the difference between average signal and average background images.

\section{Experiments}

A standard measurement was performed at 7 occasions during a period of six months to assess the reproducibility of $\mathrm{SNR}^{2}$ rate. This measurement was done using a $2 \mathrm{~mm}$ copper filter at the collimator, normal dose rate, a pulse rate of $15 \mathrm{~s}^{-1}, 0.3 \mathrm{~mm}$ additional copper filter, $42 \mathrm{~cm}$ field of view and collimated to a beam area of $400 \mathrm{~cm}^{2}\left(20 \times 20 \mathrm{~cm}^{2}\right)$. With the first four occasions, a $512^{2}$ image matrix was used whereas with the last three a $1024^{2}$ matrix was used. 
To study how the $S N R_{\text {rate }}^{2}$, the $P_{K A \text {,rate }}$ and the dose efficiency $S N R_{\text {rate }}^{2} / P_{K A \text {,rate }}$ depend on the fluoroscopy dose rate mode of operation, separate image series were acquired on the same day, using three different dose rate settings, i.e. Low (Fluoro-), Medium (Fluoro N) and High (Fluoro+). To study the effect of the pulse rate on the $S N R_{\text {rate }}^{2}$, the $P_{K A \text {,rate }}$ and the dose efficiency ( $\left.S N R_{\text {rate }}^{2} / P_{K A, \text { rate }}\right)$, separate image series were also acquired on the same day, using 10 pps, 15 pps and 30 pps. Finally, to study the effect of varying the field of view on the $S N R_{\text {rate }}^{2}$, the $P_{K A, \text { rate }}$ and the dose efficiency $\left(S N R_{\text {rate }}^{2} / P_{K A, \text { rate }}\right)$, image series were acquired on the same day, using $16 \mathrm{~cm}, 32 \mathrm{~cm}$ and $42 \mathrm{~cm}$ fields of view. When acquiring image series at different pulse rates, a $512^{2}$ image matrix was used. When acquiring image series at different dose rates and fields of view, a $1024^{2}$ image matrix was used, and all other parameters were identical to those of the standard measurement.

\section{$\mathrm{SNR}^{2}$ rate measurements and analysis}

In signal detection theory, image quality can be quantified by a model observer acting on, or analyzing, the images. The model observer computes a decision variable $D(g \mid s)$. This variable correlates with the confidence of the observer that the detail is present or absent in the image $g$, where $s$ is either of the two image data sets 'signal' or 'background'. A model observer template is derived from the difference of the average signal and background images, respectively.

The model observer used in this study has previously been described in detail ${ }^{(8-10)}$. In short, the model observer observes the individual 1024 signal and background images separately and computes the signal-to noise ratio, SNR, from the average and standard deviation in the decision variable. Each data set (signal and background) of 1024 images was divided into k 
consecutive frames $(\mathrm{k}=32)$ of $\mathrm{m}$ image sequences $(\mathrm{m}=32)$. The template $\Delta g_{D C s H F s, m}(i, j)$ (i.e. mean difference between the signal and background images) was calculated for each sequence $\mathrm{m}$, but leaving sequence $\mathrm{m}$ out of the template. This was done to avoid any bias in the model observer by using the same images to both form the model observer template and to analyze the images. The template was then cross correlated with each image frame in sequence m, $g_{s}(i, j, k, m)$ to form the DC supressing and high frequency supressing observer: "DCsHFs". The DCsHFs-observer conditional decision variables (eq.1) were needed in (eq.2)

$D_{D C s H F s}\left(g_{k, m} \mid s\right)=\sum_{i, j} \Delta g_{D C s H F s, m}(i, j) \cdot g_{s}(i, j, k, m)$

where $i$ and $j$ are pixels in the range $1 \leq i \leq 64$ and $1 \leq j \leq 64$.

The DCsHFs is similar to the ideal observer, but ignores variations (e.g. noise) in DC level (brightness variations) and noise in the highest spatial frequency. This is because these variations do not typically have much influence on the human observer. The decision is measured statistically and is described by the separability of the conditional distributions of the decision variable $D(g \mid s)$ for the two cases 'signal' and 'background'. The separation of the two cases is quantified by the difference of their two means $(D \overline{(g \mid s i g n a l)}$ $D \overline{(\text { g|background })})$ divided by the standard deviation $\sigma_{D}$ of the distributions. The model observer's signal-to noise ratio, SNR is therefore computed as

$S N R=\frac{|D \overline{(\text { g|signal })}-D \overline{(\text { g|background })}|}{\sigma_{D}}$

The $\mathrm{SNR}^{2}$ rate is computed as the $\mathrm{SNR}^{2}$ single frame multiplied by the noise lag factor, which is the number of statistically independent frames per second ${ }^{(8)}$. If no lag was present, the $\mathrm{SNR}^{2}$ rate would be $\mathrm{SNR}^{2}$ single frame multiplied by the number of frames per second. Since noise in 
adjacent frames is not independent but correlated due to lag, the increase in $\mathrm{SNR}^{2}$ rate increases more gradually with the number of frames.

\section{RESULTS}

Figure 2 shows how $S N R_{\text {rate }}^{2}, P_{K A, \text { rate }}$ and $S N R_{\text {rate }}^{2} / P_{K A, \text { rate }}$ varies with constant imaging settings during a period of 6 months. The mean $S N R_{\text {rate }}^{2} / P_{K A \text {,rate }}$ was $3.6 \pm 0.2 \cdot 10^{3} \mu \mathrm{Gy}^{-1} \mathrm{~m}^{-2}$ and the relative standard deviation from seven repeated measurement was 5.1\%. The average standard deviation ${ }^{(8)}$ in $S N R_{\text {rate }}^{2}$ was estimated to $7.3 \%$ (range 5.5-8.6\%) as indicated in tables 1-3 and the relative uncertainty in $P_{K A \text {,rate }}$ was estimated to less than $6 \%(\mathrm{k}=2)^{(7)}$. After the fourth measurement (see figure 2), the tube current increased from $26 \mathrm{~mA}$ to $34 \mathrm{~mA}$ and the $P_{K A \text {,rate }}$ increased from approximately $1.7 \mu \mathrm{Gym}^{2} / \mathrm{s}$ to $2.1 \mu \mathrm{Gym}^{2} / \mathrm{s}$. The mean $\pm 1 \mathrm{SD}$ of $S N R_{\text {rate }}^{2}$ increased in approximate proportion to dose rate from $5.9 \cdot 10^{3} \pm 0.19 \cdot 10^{3} \mathrm{~s}^{-1}$ to $7.6 \cdot 10^{3} \pm 0.10 \cdot 10^{3} \mathrm{~s}^{-1}$ leaving the ratio $S N R_{\text {rate }}^{2} / P_{K A, \text { rate }}$ approximately the same. The reason for the increase in tube current is presently not known, but it was noted that the corresponding increase in $S N R_{\text {rate }}^{2}$ was clearly detected by the method and correlated to the change in matrix size.

Table 1 shows how the $S N R_{\text {rate }}^{2}$, the $P_{K A \text {,rate }}$ and the dose efficiency $S N R_{\text {rate }}^{2} / P_{K A \text {,rate }}$ depend on the fluoroscopy dose rate mode of operation. While the $P_{K A \text {,rate }}$ increased from the low dose rate mode to the medium and high dose rate modes, the low dose rate mode yields approximately $79 \%$ and $69 \%$ higher dose efficiencies compared to the medium dose rate mode and the high dose rate mode, respectively. 
OXMI2015-026

Table 2 shows the effect of the pulse rate on the $S N R_{\text {rate }}^{2}$, the $P_{K A \text {,rate }}$ and the dose efficiency $\left(S N R_{\text {rate }}^{2} / P_{K A, \text { rate }}\right)$. The dose efficiency is approximately independent of pulse rate because $S N R_{\text {rate }}^{2}$ and $P_{K A, \text { rate }}$ both increase in proportion with pulse rate.

Table 3 shows the effect of varying the field of view from $32 \mathrm{~cm}$ to $16 \mathrm{~cm}$ on the $S N R_{\text {rate }}^{2}$, the $P_{K A, \text { rate }}$ and the dose efficiency $\left(S N R_{\text {rate }}^{2} / P_{K A, \text { rate }}\right)$. The $S N R_{\text {rate }}^{2}$ increased and $P_{K A, \text { rate }}$ decreased with decreasing field of view, which resulted in a large increase in $S N R_{\text {rate }}^{2} / P_{K A \text {,rate }}$ with decreasing field of view.

\section{DISCUSSION}

The main finding in this study was that the standard deviation in $S N R_{\text {rate }}^{2} / P_{K A, \text { rate }}$ from seven repeated measurements, performed during a period of six months was $5.1 \%$, which indicates that the method could be suitable for monitoring low-contrast detectability during quality control constancy tests in modern fluoroscopy systems.

Marshall et al. ${ }^{(1)}$ performed subjective, visual low-contrast detail evaluation measurements in fluoroscopy and found the relative standard deviation to be approximately $11 \%$ (both within and between observer variations). This corresponded to an inferior precision compared to our objective $S N R_{\text {rate }}^{2}$ measurements, but was superior to corresponding subjective visual experiments by Tapiovaara and Sandborg, who estimated the relative standard deviation (within and between observer variations) to $14 \%$ and $19 \%$ respectively ${ }^{(3)}$.

The relative standard deviation in measured low-contrast detectability found in this study was slightly larger than 3\% that was previously found with measurements of SNR using an x-ray image intensifier system ${ }^{(3)}$. While the cause of the difference is not fully known, it should be noted that both the phantom and the imaging parameters differed between our study and the 
previous study. Tapiovaara and Sandborg used a $4.7 \mathrm{~cm}$ thick PMMA phantom with an additional 2.9 mm thick PMMA disk as contrast detail, whereas we used a $5 \mathrm{~cm}$ thick PMMA phantom and a $5 \mathrm{~mm}$ thick aluminium cylinder shaped disk as contrast detail. Therefore, we would expect a higher precision in our study, since the precision of the SNR measurement is known to be worse with lower contrast detail, as a result of increased residual noise in the observer's decision template ${ }^{(8)}$. On the other hand, in the previous study, a Philips x-ray image intensifier system not used in clinical practice was used, whereas the equipment used in this work was in clinical use. Finally, the uncertainty due to small non-intentional changes in imaging geometry and imaging system output may further explain the different results found in our study and the study by Tapiovaara and Sandborg ${ }^{(3)}$.

The results of this study indicated that the low dose rate mode yields superior dose efficiency. The low dose rate mode uses a tube voltage of $87 \mathrm{kV}$ and $0.9 \mathrm{~mm}$ added $\mathrm{Cu}$ filtration compared to $81 \mathrm{kV}$ and 0.2 and $0.3 \mathrm{~mm} \mathrm{Cu}$ in the high and medium dose rate modes, respectively. The air kerma area rate for a given air kerma rate at the image detector is typically reduced with increasing tube voltage and added $\mathrm{Cu}$ filtration as the photons that contribute mostly to air kerma are those of lower energy where the linear energy absorption coefficient for air $\mu_{\text {en,air }}$ is larger. However, if an increase in absolute image quality $\left(S N R_{\text {rate }}^{2}\right)$ is required then the high dose rate mode is needed in spite of its lower dose-efficiency.

We found that with a reduction of the field of view from $32 \mathrm{~cm}$ to $16 \mathrm{~cm}$, the $S N R_{\text {rate }}^{2}$ increased by a factor of 1.7 due to the typical increase in air kerma rate (because of a change in tube current from $52 \mathrm{~mA}$ to $95 \mathrm{~mA}$ ) associated with a reduction in field of view. Since the x-ray beam area is approximately reduced to $25 \%$ with this change in field of view, the reduction in $P_{K A, \text { rate }}$ is approximately $50 \%$. The dose efficiency $\left(S N R_{\text {rate }}^{2} / P_{K A, \text { rate }}\right)$ increased by more than a factor of 3 . 
While the results of this study showed that measurements of $S N R_{\text {rate }}^{2} / P_{K A, \text { rate }}$ are promising for use in quality control constancy tests, we encountered some practical limitations that were mainly related to the large size of the image stacks (1024 images) produced by the fluoroscopy equipment, in particular with an image matrix size of $1024^{2}$ pixels. The transfer of the image stacks to the PACS, and the subsequent export of the stacks to the analysis software was sometimes slow and prone to errors. Therefore, future studies are needed to investigate the effect of reducing the number of frames and reducing the matrix size on the measurement precision. Although the contrast objects used in this study (aluminium discs) are useful in quality control constancy tests, further studies could focus on finding contrast objects that are more clinically relevant. These may also include contrast agents such as iodine, which are commonly used during fluoroscopy procedures. This may ultimately make $S N R_{\text {rate }}^{2} / P_{K A, \text { rate }}$ measurements a useful method for patient dose optimisation.

\section{CONCLUSION}

Our results show that $S N R_{\text {rate }}^{2} / P_{K A, \text { rate }}$ measurements offer the high precision that is needed in quality control constancy tests and that these measurements make it possible to identify dose-efficient imaging configurations on a modern fluoroscopy system.

\section{FUNDING}

This work was supported by ALF (Avtal om Läkarutbildning och Forskning) grants from Region Östergötland 2014 [LIO-357651]. 
OXMI2015-026

\section{ACKNOWLEDGMENTS}

The Department of Radiology at Linköping University hospital is acknowledged for accessing their x-ray unit during daytime.

\section{REFERENCES}

1. Marshall, N.W., Faulkner, K., Cotre, C.J. and Robson, K. Analysis in variation in contrast detail measurements performed on image intensifier television system. Phys. Med. Biol., 37, 2297-2302 (1992).

2. Burgess, A.E. Comparison of receiver operating characteristics and forced choice observer performance methods. Med. Phys., 22, 643-655 (1995).

3. Tapiovaara, M.J. and Sandborg, M. How should low-contrast detail detectability be measured in fluoroscopy? Med. Phys. 31, 2564-2576 (2004).

4. Thilander-Klang, A., Ledenius, K., Hansson, J., Sund, P. and Båth, M. Evaluation of subjective assessment of the low-contrast visibility in constancy control of computed tomography. Radiat. Prot. Dosim.139, 449-454 (2010).

5. Racine, D., Ba, A.H., Ott, J.G., Ryckx, N., Bochud, F.O., and Verdun, F.R. Objective task based assessment of low contrast detectability in iterative reconstruction. Radiat. Prot. Dosim. (this issue).

6. Ott, J.G., Ba, A.H., Racine, D., Ott, J.G., Ryckx, N., Bochud, F.O., Alkadhi, H. and Verdun F.R. Patient exposure optimisation through task-based assessment of a new iterative reconstruction technique. The ADMIRE algorithm. Radiat. Prot. Dosim. (this issue).

7. Malusek, A., Sandborg, M. and Alm Carlsson, G. Accurate KAP-meter calibration as a prerequisite for optimisation in projection radiography. Radiat. Prot. Dosim. (this issue)

8. Tapiovaara, M. Objective measurement of image quality in fluoroscopy x-ray equipment: FluoroQuality. STUK-A196, STUK, Radiation and Nuclear Safety Authority, Helsinki, Finland (2003). ISBN 951-712- 688-1

9. Tapiovaara, M.J. SNR and noise measurements for medical imaging: II. Application to fluoroscopic x-ray equipment. Phys. Med. Biol. 38, 1761-1788 (1993). 
OXMI2015-026

10. Tapiovaara, M.J. Efficiency of low-contrast detectability in fluoroscopy imaging. Med. Phys., 24, 655-664 (1997). 
OXMI2015-026

LEGENDS TO TABLES

\section{Table 1}

The effect of dose rate mode on $S N R_{\text {rate }}^{2}, P_{K A \text {,rate }}$ and $S N R_{\text {rate }}^{2} / P_{K A, \text { rate }}$. SNR $\mathrm{S}_{\text {rate }}$ : Signal-to noise ratio rate, $\mathrm{P}_{\mathrm{KA}, \mathrm{rate}}$ : air kerma area product rate.

\section{Table 2}

The effect of pulse rate on $S N R_{\text {rate }}^{2}, P_{K A \text {,rate }}$ and $S N R_{\text {rate }}^{2} / P_{K A \text {,rate }} . \mathrm{SNR}_{\text {rate }}$ : Signal-to noise ratio rate, $\mathrm{P}_{\mathrm{KA}, \text { rate: }}$ air kerma area product rate.

\section{Table 3}

The effect of field of view on $S N R_{\text {rate }}^{2}, P_{K A, \text { rate }}$ and $S N R_{\text {rate }}^{2} / P_{K A, \text { rate }} . \mathrm{SNR}_{\text {rate: }}$ Signal-to noise ratio rate, $\mathrm{P}_{\mathrm{KA} \text {,rate: }}$ air kerma area product rate. 
OXMI2015-026

TABLES

Table 1

$$
\begin{array}{rrr}
\text { SNR }^{2} \text { rate } & \mathbf{P}_{\text {KA,rate }} & \mathbf{S N R}^{2} \text { rate } / \mathbf{P}_{\text {KA,rate }} \\
\left(\mathrm{s}^{-1}\right) & \left(\mu \mathrm{Gym}^{2} \mathrm{~s}^{-1}\right) & \left(\mu \mathrm{Gy}^{-1} \mathrm{~m}^{-2}\right)
\end{array}
$$

Dose rate setting

$\begin{array}{lccc}\text { Low (Fluoro-) } & 3.6 \pm 0.20 \cdot 10^{3} & 0.56 & 6.45 \pm 0.36 \cdot 10^{3} \\ \text { Medium (Fluoro N) } & 7.5 \pm 0.57 \cdot 10^{3} & 2.08 & 3.61 \pm 0.27 \cdot 10^{3} \\ \text { High (Fluoro+) } & 13.9 \pm 0.80 \cdot 10^{3} & 3.60 & 3.87 \pm 0.22 \cdot 10^{3}\end{array}$

Table 2

\begin{tabular}{cccc} 
& $\begin{array}{c}\mathbf{S N R}^{2} \text { rate } \\
\left(\mathrm{s}^{-1}\right)\end{array}$ & $\begin{array}{c}\mathbf{P}_{\mathrm{KA}, \text { rate }} \\
\left(\mu \mathrm{Gym}^{2} \mathrm{~s}^{-1}\right)\end{array}$ & $\begin{array}{c}\mathbf{S N R}^{2} \text { rate } \\
\left(\mu \mathbf{P}_{\mathrm{KA}, \text { rate }} \mathrm{m}^{-2}\right)\end{array}$ \\
\hline Pulse rate & & & \\
$10 \mathrm{pps}$ & $4.1 \pm 0.29 \cdot 10^{3}$ & 1.16 & $3.52 \pm 0.25 \cdot 10^{3}$ \\
$15 \mathrm{pps}$ & $5.8 \pm 0.40 \cdot 10^{3}$ & 1.72 & $3.35 \pm 0.23 \cdot 10^{3}$ \\
$30 \mathrm{pps}$ & $12.6 \pm 0.88 \cdot 10^{3}$ & 3.43 & $3.67 \pm 0.26 \cdot 10^{3}$ \\
\hline
\end{tabular}

Table 3

$$
\text { SNR }^{2} \text { rate } \quad \mathbf{P}_{\text {KA,rate }} \text { SNR }^{2} \text { rate } / \mathbf{P}_{\text {KA,rate }}
$$

$\left(\mathrm{s}^{-1}\right) \quad\left(\mu \mathrm{Gym}^{2} \mathrm{~s}^{-1}\right) \quad\left(\mu \mathrm{Gy}^{-1} \mathrm{~m}^{-2}\right)$

\section{Field of view}

$\begin{array}{llll}32 \mathrm{~cm} & 11.0 \pm 0.93 \cdot 10^{3} & 2.40 & 4.58 \pm 0.39 \cdot 10^{3} \\ 22 \mathrm{~cm} & 17.3 \pm 1.49 \cdot 10^{3} & 1.82 & 9.52 \pm 0.82 \cdot 10^{3} \\ 16 \mathrm{~cm} & 18.8 \pm 1.26 \cdot 10^{3} & 1.31 & 14.38 \pm 0.96 \cdot 10^{3}\end{array}$


OXMI2015-026

\section{LEGENDS TO FIGURES}

Figure 1. Fluoroscopy images of the inhomogeneous phantom containing the $5 \mathrm{~mm} \mathrm{Al}$ contrast detail that was used for the calculations of $S N R_{\text {rate }}^{2}$. Single signal image frame $g_{1}$ (left), single background image frame $g_{0}$ (middle) and the average difference image $\overline{\Delta g}=$ $\overline{g_{1}}-\overline{g_{0}}$ i.e. the template (right).

Figure 2. The $S N R_{\text {rate }}^{2}$, the $P_{K A \text {,rate }}$ and the $S N R_{\text {rate }}^{2} / P_{K A \text {,rate }}$ for repeated measurements with the same imaging conditions (apart from the matrix size) during a period of six months. The estimated uncertainty is indicated by the error bars. The relative standard deviation in the $S N R_{\text {rate }}^{2} / P_{K A \text {,rate }}$ from repeated measurements was 5.1\%. The dashed vertical line indicates a change in tube current (and matrix size) that was observed between the fourth and fifth measurement, which notably affected the $S N R_{\text {rate }}^{2}$ and the $P_{K A \text {,rate }}$, but not the $S N R_{\text {rate }}^{2} / P_{K A, \text { rate }}$ 
OXMI2015-026

FIGURES

Figure 1.
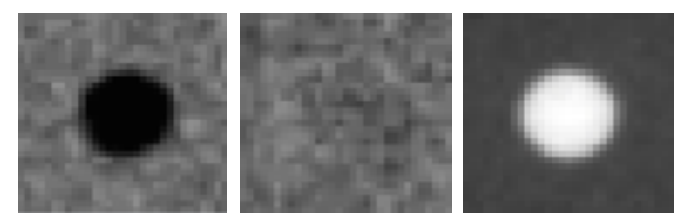

Figure 2.

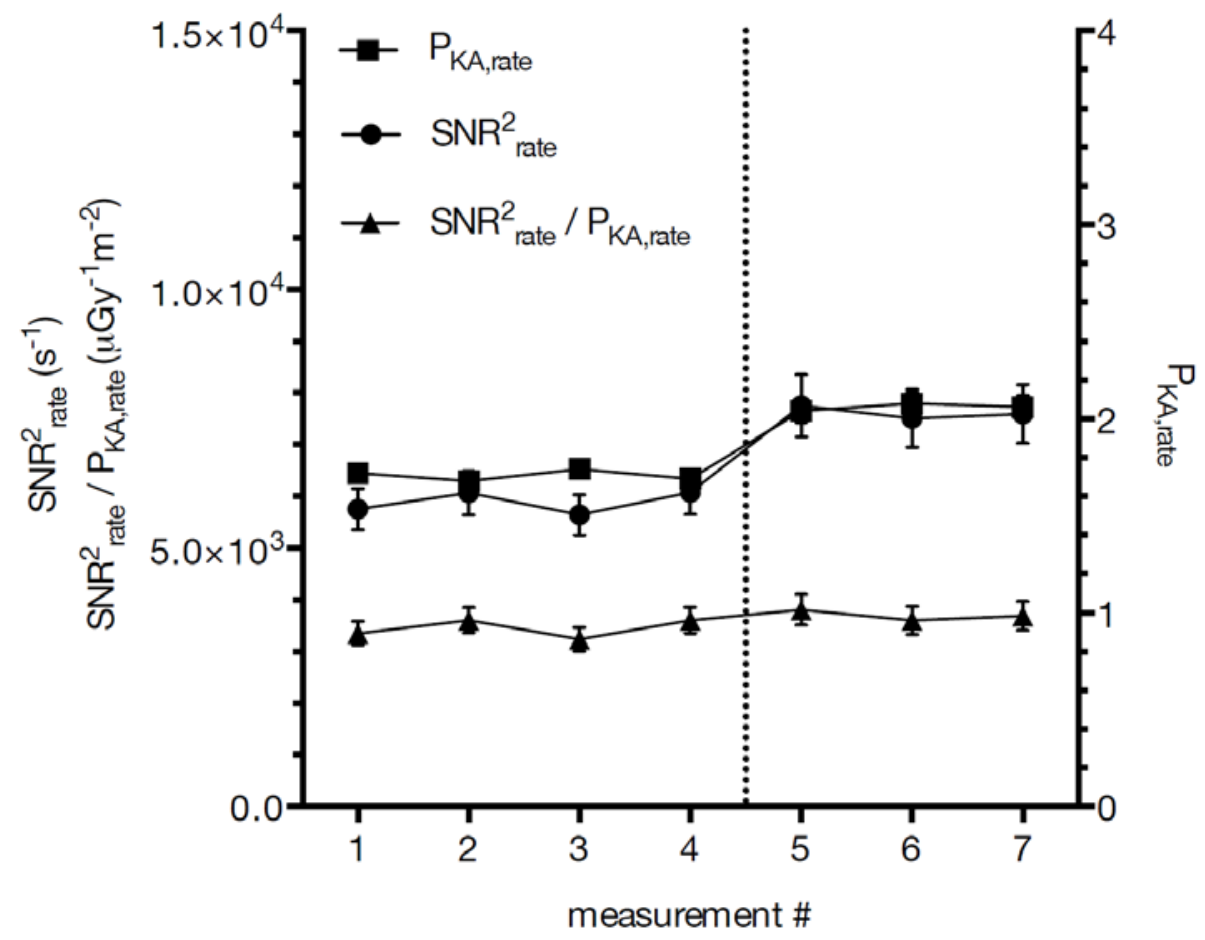

